\title{
IN VITRO ANTIBACTERIAL ACTIVITY OF MENTHA ESSENTIAL OILS AGAINST STAPHYLOCOCCUS AUREUS
}

\author{
Horváth, P., Koščová, J. \\ Department of Microbiology and Immunology \\ University of Veterinary Medicine and Pharmacy \\ Komenského 73, 04181 Košice \\ Slovakia
}

jana.koscova@uvlf.sk

\section{ABSTRACT}

Plant extracts and essential oils (EOs) are characterized by their antibacterial properties against various bacterial pathogens, including staphylococci. Some strains of these bacteria are resistant against the adverse effects of the environment including antibiotics, e.g. methicillin-resistant Staphylococcus aureus (MRSA). EOs alone cannot substitute for antibiotics but their treatment may be useful to intensify and strengthen the effects of antibiotics on pathogenic staphylococci. In this work, we tested the antibacterial effects of the essential oils of Mentha species with menthol as one of the effective substances against different strains of $S$. aureus. Two in vitro methods were used, the qualitative disc diffusion assay and the quantitative minimal inhibitory concentration (MIC) of selected essential oils. Peppermint oil from Mentha piperita, spearmint oil from Mentha spicata var. crispa and cornmint oil from Mentha arvensis were tested in this study against the various strains of Staphylococcus aureus, including methicillin resistant Staphylococcus aureus (MRSA). The oils were dissolved in DMSO (dimethyl sulfoxide) and diluted at the following ratios: $1: 1,1: 2,1: 5$, and $1: 10$. Based on the results determined by the agar disc diffusion test, the highest antibacterial properties were observed in spearmint oil against $S$. $a u$ reus CCM 4223 at 1:2 ratio where the inhibition zone varied at a range of $35.67 \pm 6.81 \mathrm{~mm}$. We determined also the MIC of all the oils where concentrations of the oils were as follows: $1 \% ; 0.5 \% ; 0.25 \% ; 0.125 \%$ and $0.0625 \%$. The lowest concentrations of essential oils that possessed inhibitory effects on the growth of S.aureus varied between $0.125 \%$ and $0.25 \%$.

Key words: antibacterial activity; essential oils; in vitro methods; Staphylococcus aureus

\section{INTRODUCTION}

The essential oils (EOs) are a group of various natural chemicals which are characterized by their volatility and aroma. The term "essential oil" comes from the times of the Middle Ages. At this time, alchemists considered every 
liquid floating on the water as an oil. After the invention of the distillation procedure, they thought that EOs are "the essence of life".

The plants produce EOs for their protection against herbivores. EOs can concentrate in a specific plant organ or they pervade the whole plant (conifers). Naturally they are colourless. At room temperature the majority of EOs occurs in liquid form; some are solids (camphora). Mostly they have lower density than water, with the except of cinnamon and clove oils. They dissolve in alcohol and fats. The main chemical compounds in EOs are terpenes-monoterpenes (ocimene, limonene, linalool, $\alpha$-pinene) and sesquiterpenes ( $\beta$-caryophyllene). More complex terpenes have higher molecular weights and because of that, they cannot be distilled. Other chemical groups are alcohols (geraniol, menthol), aldehydes (cinnamaldehyde, vanillin), ketones (carvone), and phenols (eugenol, thymol, carvacrol) [12].

EOs have a wide range of applications. They can be used as antiflogistics, stomachics, carminatives, diuretics, sedatives, antimycotics, antivirotics, disinfectants, etc. One of the main benefit is also their antibacterial effects. EOs act by various mechanism on different bacterial structures. The structure of gram-positive bacteria facilitates the penetration of hydrophobic molecules into the cell and act on the bacterial wall, cytoplasmic membrane or cytoplasm. At low concentrations, they can react with enzymes responsible for producing energy, at higher concentration they can denaturate proteins. Because of the reduced proton gradient by influencing the transfer of $\mathrm{H}^{+}$, essential oils reduce the synthesis of adenosine triphosphate (ATP) and thus the intracellular store of ATP. They can cause the degradation of bacterial cell walls, damage of cytoplasmic membranes and coagulation of the cytoplasm. By damaging the membrane proteins, they increase the permeability of the membrane and cause leakage of the cell contents [4]. In general, gramnegative $\left(\mathrm{G}^{-}\right)$bacteria are more resistant against EOs in comparison to gram-positive $\left(\mathrm{G}^{+}\right)$because of the different composition of the bacterial cell walls [15]. G- bacteria have a thin layer of peptidoglycan and lipopolysaccharide layer (LPS) on their outer membrane that is lacking in grampositive bacteria. Small hydrophilic molecules can penetrate through the porin proteins of $\mathrm{G}^{-}$bacteria. The porins are relatively resistant to hydrophobic molecules, but not completely. Some EOs, e.g. from basil, sage or oregano, act on E. coli, S. aureus, B. cereus and Salmonella spp. However, they are less effective against Pseudomonas spp. because it increases its resistency by producing exopolysaccharides and by creating biofilms [7].

Bacteria of the species Staphylococcus aureus are Grampositive, facultative anaerobic and non-motile cocci. They cause many diseases in humans and animals that can be local or even systemic. Staphylococci can be found on the skin, skin wounds or abrasions. The infection can be spread by hands or by secretion from the nose or mouth. Some S. aureus strains produce at least 11 enterotoxins designated SEA to SEJ. Toxin types A and D are most frequently implicated in the outbreaks of food poisoning. These toxins are resistant to a temperature of $100^{\circ} \mathrm{C}$ within 20 minutes. Bacteria are cold resistant, while heating over $60^{\circ} \mathrm{C}$ will killing them [1]. An intoxication called staphyloccocal enterotoxicosis results from contaminated food where the bacteria are spreading and producing the toxins. Pathogenic staphylococcal strains can cause also pneumonia, post-operative infections and nosocomial bacteremia [13]. Methicillin resistant Staphylococcus aureus (MRSA) strains are dangerous, because infections caused by these strains cannot be treated with many antibiotics (ATB), as they are multiresistant. The resistance against ATB is caused by the overuse of ATB or inappropriate dosage. Furthermore, many MRSA strains produce biofilms. The biofilm is a product of the microbial population attached on the substrate or on to another. It is a polymeric substance that arises due to a changed bacterial fenotype related to its growth, gene expression and synthesis of proteins. The production of biofilm is strictly regulated by genetic factors. Immune response against the biofilm infections is uneffective and leads to chronic diseases [2].

Some of the more popular methods used for testing antimicrobial activity of EOs is the disc diffusion method, the determination of minimum inhibitory concentration (MIC) and the vapour phase method. Additional methods are bioautography TLC method for testing the antimicrobial activity of individual components of EOs [3].

The purpose of this study was to investigate the antibacterial properties of three various species of the mint essential oils and menthol against Staphylococcus aureus strains using two in vitro methods.

\section{MATERIALS AND METHODS}

\section{Bacterial strains}

All together 4 strains of Staphylococcus aureus were 
used in this study: S.aureus (clinical isolate from a dog wound), S.aureus CCM 4750 (MRSA) and 2 strains of S. aureus with the ability to produce biofilms (CCM 3953 and CCM 4223). The strains with CCM numbers were obtained from the Czech Collection of Microorganisms in Brno (Czechia).

\section{Essential oils}

Three different species of the mint essential oils and menthol were tested in this study. Menthaxpiperita L. peppermint oil (Calendula a.s., Nová Lubovňa) with its mayor components L-menthol (40.7\%) and menthone (23.4\%). Mentha spicata L. var. crispa - spearmint oil (Hanus s.r.o., Nitra) with its mayor components L-carvone (70\%) and limonene (10\%). Mentha arvensis L. - cornmint (Hanus s.r.o., Nitra) with its mayor components Lmenthol (28.8-34.7\%), menthone (16.3-1.1\%) and isomenthone $(6.8-12.1 \%)$. And finally, we used also a pure component, L-menthol (Galvex, s.r.o.).

\section{Growth conditions}

Growth media: PYG broth (Pepton Yeast Extract Glucose Broth) was used for overnight cultivation of the bacterial strains. MHA (Mueller Hinton Agar, HiMedia, India) was used for the disc diffusion method and also for the MIC determinations.

The preparation of the overnight culture: $3 \mathrm{ml}$ of PYG broth was inoculated with the various staphylococcal strains and incubated on a shaker at $37^{\circ} \mathrm{C}$ for $18 \mathrm{~h}$.

Determination of CFU (colony forming units): After incubation, the counts of bacteria in overnight culture were determined. The results for each strain are shown in Table 1.

\section{Disc diffusion method}

Table 1. Number of CFU.ml-1 after $18 \mathrm{~h}$ incubation at $37^{\circ} \mathrm{C}$

\begin{tabular}{ll}
\hline \multicolumn{1}{c}{ Bacterial strain } & CFU.ml $^{-1}$ \\
\hline Staphylococcus aureus - clinical dog isolate & $3.32 \times 10^{11}$ \\
Staphylococcus aureus (MRSA) CCM 4750 & $3.16 \times 10^{12}$ \\
Staphylococcus aureus CCM 3953 & $3.31 \times 10^{12}$ \\
Staphylococcus aureus CCM 4223 & $9.4 \times 10^{10}$ \\
\hline
\end{tabular}

This method was used as qualitative method to determine the antimicrobial activity of selected essential oils. $0.1 \mathrm{ml}$ of overnight culture was spread onto the surface of MHA using a sterile bacteriological spreader. After the absorption of inoculum, we transferred the paper discs (6 mm, Becton, Dickinson and Company, USA) with sterile needles onto the surface of the inoculated MHA agar and applied with a micropipette $10 \mu \mathrm{l}$ of each EO in several dillutions $(1: 1,1: 2,1: 5,1: 10)$ onto the paper discs. Pure concentrated DMSO (dimethyl sulfoxide) was used as a negative control and specific ATBs (oxacillin $5 \mu \mathrm{g} / \mathrm{disc}$ and methicillin $5 \mu \mathrm{g} / \mathrm{disc}$ ) were used as positive controls. The Petri dishes were incubated for $24 \mathrm{~h}$ at $37^{\circ} \mathrm{C}$. After the incubation time, the diameter of the inhibition zones (in $\mathrm{mm}$ ) around the paper discs were measured. This in vitro test was repeated in triplicate to get an average number with the standard deviation as a result.

\section{Determination of MIC}

(Minimal Inhibitory Concentration)

Two-fold dilutions of EOs with concentrations of $1 \%$, $0.5 \%, 0.25 \%, 0.125 \%$ and $0.0625 \%$ in MHA with $0.5 \%$ Tween 20 (Sigma Aldrich, Germany) were prepared before starting the test. Two $\mu$ l of overnight culture were inoculated with a micropipette drop by drop onto the surface of MHA [11]. Medium without EOs was used as a negative control. The Petri dishes were incubated for $24 \mathrm{~h}$ at $37^{\circ} \mathrm{C}$. The experiments were carried out in triplicate. We determined the MIC as a minimal concentration of the EOs that suppressed the growth of bacteria on the agar dishes.

\section{Statistics}

The results obtained by the disc diffusion method are reported as an arithmetical average \pm standard deviation in $\mathrm{mm}$. We considered $\mathrm{d}>6 \mathrm{~mm}$ as a positive result. The statistic software GraphPad Prism version 3 was used and the results were evaluated by one-way ANOVA and Tukey's test with ${ }^{* * *} \mathrm{P}<0.001$ as a significance level. The results obtained by the MIC method were evaluated only as presence/absence of growth of bacteria on the surface of the agar plate.

\section{RESULTS AND DISCUSSION}

\section{Disc diffusion method}

The evaluation of antibacterial activity of mint essential oils showed that they effectively inhibited the staphylococcal strains, hovewer, with different sensitivities. 
Table 2. Clinical isolate Staphylococcus aureus, disc diffusion method $(n=3)$, inhibition zones [mm]

\begin{tabular}{cccccc}
\hline Dilution & M.piperita & M. arvensis & M.spicata & L-menthol & $\begin{array}{c}\text { Oxacillin } \\
\mathbf{5} \boldsymbol{\mu g} / \mathbf{d i s c}\end{array}$ \\
\hline $1: 10$ & $2.33 \pm 4.04$ & $7 \pm 0$ & $0 \pm 0^{* * *}$ & $7.5 \pm 0$ & 22 \\
$1: 5$ & $9 \pm 0^{* * *}$ & $9.33 \pm 0.58^{* * *}$ & $9.33 \pm 0.58^{* * *}$ & $15.67 \pm 4.04$ & 24 \\
$1: 2$ & $18.17 \pm 1.26$ & $15.33 \pm 2.52$ & $22.33 \pm 2.31$ & $20.5 \pm 0.87$ & 23 \\
$1: 1$ & $15.5 \pm 0.87$ & $16.67 \pm 1.16$ & $15 \pm 2$ & $15.17 \pm 0.29$ & 21 \\
\hline
\end{tabular}

*** — significantly different from Oxacillin $(P<0.001)$

Table 3. Methicillin resistant S. aureus (MRSA) CCM 4750, disc diffusion method $(n=3)$, inhibition zones [mm]

\begin{tabular}{cccccc}
\hline Dilution & M. piperita & M.arvensis & M. spicata & L-menthol & $\begin{array}{l}\text { Oxacillin } \\
\mathbf{5} \boldsymbol{\mu g} / \mathbf{d i s c}\end{array}$ \\
\hline $1: 10$ & $0 \pm 0$ & $0 \pm 0$ & $0 \pm 0$ & $0 \pm 0$ & 22 \\
$1: 5$ & $5 \pm 4.33$ & $4.67 \pm 4.04$ & $0 \pm 0$ & $8 \pm 0$ & 24 \\
$1: 2$ & $10.67 \pm 1.53$ & $10.67 \pm 2.02$ & $11.33 \pm 9.87$ & $12 \pm 1$ & 23 \\
$1: 1$ & $11.5 \pm 0.5$ & $13.83 \pm 3.62$ & $9.33 \pm 0.29$ & $10.67 \pm 0.29$ & 21 \\
\hline
\end{tabular}

Table 4. Staphylococcus aureus CCM 3953, disc diffusion method ( $n=3)$, inhibition zones [mm]

\begin{tabular}{cccccc}
\hline Dilution & M. piperita & M. arvensis & M. spicata & L-menthol & $\begin{array}{c}\text { Oxacillin } \\
5 \mu \mathrm{g} / \mathrm{disc}\end{array}$ \\
\hline $1: 10$ & $0 \pm 0^{\star * *}$ & $0 \pm 0^{\star * *}$ & $0 \pm 0^{\star * *}$ & $2.5 \pm 4.33^{\star * *}$ & 15 \\
$1: 5$ & $7.67 \pm 0.29^{* * *}$ & $7.5 \pm 0.5^{\star * *}$ & $6.5 \pm 0^{\star * *}$ & $9.5 \pm 1.32^{\star * *}$ & 18 \\
$1: 2$ & $17 \pm 1$ & $15.83 \pm 2.02$ & $15.33 \pm 0.58$ & $14.67 \pm 1.61$ & 19 \\
$1: 1$ & $12.33 \pm 1.53$ & $12.33 \pm 1.44$ & $10.83 \pm 0.76$ & $12.5 \pm 0.5$ & 14 \\
\hline
\end{tabular}

*** - significantly different from Oxacillin $(P<0.001)$

Table 5. Staphylococcus aureus CCM 4223, disc diffusion method ( $\mathrm{n}=3$ ), inhibition zones [mm]

\begin{tabular}{|c|c|c|c|c|c|}
\hline Dilution & M. piperita & M. arvensis & M. spicata & L-menthol & $\begin{array}{c}\text { Methicillin } \\
5 \mu \mathrm{g} / \text { disc }\end{array}$ \\
\hline $1: 10$ & $0 \pm 0^{a, b}$ & $0 \pm 0^{a, b}$ & $0 \pm 0^{a, b}$ & $7.83 \pm 0.29^{b}$ & 20 \\
\hline $1: 5$ & $18 \pm 2$ & $10.67 \pm 1.26$ & $12.67 \pm 1.53$ & $14.33 \pm 2.08$ & 15 \\
\hline $1: 2$ & $22.83 \pm 0.76$ & $17.67 \pm 0.58^{c}$ & $35.67 \pm 6.81^{a, b}$ & $18 \pm 1$ & 18 \\
\hline $1: 1$ & $14.17 \pm 0.76$ & $15.5 \pm 0.5^{\mathrm{a}}$ & $16.33 \pm 0.58^{a}$ & $12.33 \pm 0.29$ & 14 \\
\hline
\end{tabular}

a — significantly different from L-menthol; ${ }^{b}$ — significantly different from Methicillin $c$ - significantly different from Mentha spicata $(P<0.001)$ 
The results of individual dilutions $1: 1,1: 2,1: 5,1: 10$ are shown in Tables $2-5$. The strongest effect was obtained with Mentha spicata var. crispa and the highest resistance was shown by the MRSA strain. It was interesting that almost in every experiment the concentration $1: 1$ exhibited lower efficiency in comparison to the concentration of $1: 2$. We used L-menthol which exhibited the major efficiency. L-menthol was used at the same volume as EOs. The best reflection of the effects of L-menthol would be the same volume as in the essential oils. But in some EOs, menthol did not represent the major part.

Significant differences were detected between Mentha spicata var. crispa and menthol and the positive control $\left({ }^{* * *} \mathrm{P}<0.001\right)$.

\section{Determination of MIC}

The concentrations which inhibited the growth of staphylococci varied between $0.125 \%$ and $0.25 \%$. The strongest effects against the MRSA was observed for M. spicata at the concentration of $0.125 \%$. M. arvensis was effective at the same concentration against both biofilm producing staphylococcal strains (CCM 3953 and CCM 4223) (Table 6).

Table 6. Minimal inhibitory concentration (MIC) [mm]

\begin{tabular}{lcccc}
\hline & S. aureus & $\begin{array}{c}\text { MRSA } \\
\text { CCM 4750 }\end{array}$ & $\begin{array}{c}\text { S. aureus } \\
\text { CCM 3953 }\end{array}$ & $\begin{array}{c}\text { S. aureus } \\
\text { CCM 4223 }\end{array}$ \\
\hline M. piperita & $0.25 \%$ & $0.25 \%$ & $0.25 \%$ & $0.25 \%$ \\
M. arvensis & $0.25 \%$ & $0.25 \%$ & $0.125 \%$ & $0.125 \%$ \\
M. spicata & $0.25 \%$ & $0.125 \%$ & $0.25 \%$ & $0.25 \%$ \\
\hline
\end{tabular}

*** $-P<0.001$

The antibacterial activity of the essential oils is a topic for many investigations. We observed antibacterial activity of EOs for 3 different species of Mentha sp. gender and Lmenthol against 4 different strains of Staphylococcus aureus.

Using the disc diffusion method, we observed that the highest antibacterial effect was achieved mostly at the concentration of $1: 2$, not $1: 1$ as expected. EO from Mentha spicata var. crispa showed the strongest activity against the clinical isolate of $S$. aureus at a concentration of $1: 2$. On the other hand, the concentration of $1: 1$ was the strongest in the case of EO from M. arvensis. The biggest diameter of inhibition zones against MRSA were measured with EO from M. spicata at the concentration of $1: 2$ and at the concentration of $1: 1$ with EO from M. arvensis. We tested also 2 spe- cies of staphylococcal strains producing biofilms. S. aureus CCM 3953 was inhibited by EO from M.piperita at the dilution of $1: 2$, S.aureus CCM 4223 by EO from M.spicata var. crispa at the same dilution.

For MIC determination we exposed all 4 strains of S. aureus to 5 different concentrations of EOs: $1 \%, 0.5 \%$, $0.25 \%, 0.125 \%$ and $0.0625 \%$. The difference in the sensitivity within staphylococci strains was not so noticeable in comparison to the disc diffusion method. The results varied between $0.125 \%$ and $0.25 \%$. The biofilm producing staphylococci CCM 3953 and CCM 4223 were the most sensitive to EO from Mentha arvensis. These results were affected by the emulgation of the EOs in agar. For better EOs homogenization, Tween 20 at $0.5 \%$ concentration was used. It appears desirable to use different concentrations of an emulgator and to compare the results.

In another study it was found that EO from M.spicata var. crispa also affected S. aureus. The sizes of the inhibition zones in the disc diffusion method were $10 \mathrm{~mm}$ at S. aureus ATCC 6538 and $8 \mathrm{~mm}$ at S. aureus ATCC 25923 despite the application of double dose $(20 \mu \mathrm{l})$ of $100 \%$ EO in comparison to a dose $10 \mu \mathrm{l}$ used in this study [10]. The reason for the different results may be caused by the different composition of EOs. S.aureus ATCC 6538 was inhibited at $0.1 \%$ concentration compared to our $0.25 \%$. In another study, it was observed that Mentha piperita also had inhibitory effects on S.aureus (the strain was not specified) [9]. Using the disc diffusion method, the measured inhibition zone was $7.6 \pm 0.57 \mathrm{~mm}$ at $1: 10$ concentration. No inhibition zone was observed in our study.

I $\mathrm{m}$ a i et al. [6] confirmed the effectivity of all three species of mint against a MRSA strain.

The results of the antimicrobial activity of essential oils can be influenced also by various extraction methods and extracts used for the determination. Para et al. [8] confirmed that among ethanol, methanol, ethyl acetate, chloroform, hexane and petroleum ether, the ethyl acetate leaf extract of Mentha piperita caused more pronounced inhibition of Staphylococcus aureus, Bacillus subtilis and Proteus vulgaris than chloroform, petroleum ether or hexane extracts.

The antibacterial activity of $M$. spicata was confirmed also in the study by Golestan et al. [5]. They observed that M.spicata EO had the highest inhibition activity against S. aureus and Clostridium perfringens.

Zaidi and Dahiya [14] used the agar well difusion method for the determination of the antimicrobial activity 
of EOs from Mentha spicata and Mentha piperita against 11 bacterial and 4 fungal clinical isolates. They reported that both of the EOs showed the maximum activity against S.aureus, producing a maximum zone of inhibition of $21 \pm 0.09 \mathrm{~mm}$ with Mentha spicata and $19.2 \pm 0.07 \mathrm{~mm}$ with Mentha piperita.

\section{CONCLUSIONS}

This study tested the antibacterial activity of EOs from Mentha sp. and L-menthol by means of the disc diffusion method and the determination of MIC. All three EOs had various contents of menthol and exhibited different effects. In spearmint oil, the main ingredient was L-carvone and not menthol.

The disc diffusion method revealed the strongest effect of EO from Mentha spicata var. crispa and the highest resistance of the MRSA strain. The EO from M. spicata var. crispa had the strongest effect against S. aureus CCM 4223. We noticed that almost in every experiment the $1: 1$ dilution exhibited lower efficiency than the $1: 2$ dilution. At the 1:2 dilution, EO from Mentha spicata var. crispa exhibited stronger or similar effect as the oxacillin/methicillin that were used as positive controls. The effect of EO from Mentha piperita was stronger against S. aureus CCM 3953.

The values of MIC varied between $0.125 \%$ and $0.25 \%$. The strongest effect against MRSA showed EO from M. spicata var. crispa that inhibited the growth of bacteria even at $0.125 \%$ concentration. M. arvensis had the strongest effect on the biofilm forming staphylococci also at this low concentration $(0.125 \%)$.

The results from our study indicated that the mint essential oils can be used as a potential source of natural antimicrobial compounds against staphylococcal infections.

\section{ACKNOWLEDGEMENT}

This study was supported by the project APVV-15-0377 and INFEKTZOON - Center of excellence for infectious disease and zoonosis ITMS code 26220120002 (operational program of research and development) financed by European Union funds for regional improvement.

\section{REFERENCES}

1. Adams, M. R., Moss, M., 2008: Food Microbiology. The Royal Society of Chemistry, $463 \mathrm{pp}$.

2. Archer, N.K., Mazaitis, M. J., Costerton, J. W., Leid, J.G., Powers, M.E., Shirtliff, M.E., 2011: Staphylococcus aureus biofilms. Properties, regulation and roles in human disease. Virulence, 2, 445-459.

3. BaşEr, K.H.C., Buchbauer, G., 2010: Handbook of Essential Oils: Science. Technology, and Applications. Boca Raton. CRC Press/Taylor, 975 pp.

4. Burt, S., 2004: Essential oils: their antibacterial properties and potential applications in foods - a review. International Journal of Food Microbiology, 94, 223-253.

5. Golestan, L., Seyedyousefi, L., Kaboosi, H., Safari, H., 2016: Effect of Mentha spicata L. and Mentha aquatica L. essential oils on the microbiological properties of fermented dairy product, kashk. Int. J. Food Sci. Technol., 51, 581-587.

6. Imai, H., Osawa, K., Yasuda, H., Hamashima, H., Arai, T., Sasatsu, M., 2001: Inhibition by the essential oils of peppermint and spearmint of the growth of pathogenic bacteria. Microbios, 106 Suppl. 1, 31-39.

7. Nazzaro, F., Fratianni, F., Martino, L., Coppola, R., De Feo, V., 2013: Effect of Essential Oils on Pathogenic Bacteria. Pharmaceuticals, 6, 1451-1474.

8. Para, S., Thulasi, M.S., Penchalaneni, J., Challagundla, V.N., 2013: Antibacterial activity and phytochemical analysis of Mentha piperita L. (Peppermint) - An important multipurpose medicinal plant. American Journal of Plant Sciences, 4, 77-83.

9. Ramos, S., Rodrigues, R. A. B. L, Farias, A. L. F. et al., 2017: Chemical composition and in vitro antioxidant, cytotoxic, antimicrobial, and larvicidal activities of the essential oil of Mentha piperita L. (Lamiaceae). The Scientific World Journal, 2017, 1-8.

10. Shahbazi, Y., 2015: Chemical composition and in vitro antibacterial activity of Mentha spicata essential oil against common food-borne pathogenic bacteria. Journal of Pathogens, $2015,1-5$.

11. Silva, F., Ferreira, S., Queiroz, J. O., Domingues, F. C, 2011: Coriander (Coriandrum sativum L.) essential oil: its antibacterial activity and mode of action evaluated by flow cytometry. Journal of Medical Microbiology, 60, 1479-1486.

12. Tomko, J., 1999: Farmacognozy (In Slovak). Textbook for pharmaceutical faculties. 2nd edn., Martin, Osveta, 424 pp. 
13. Tong, S.Y.C, Davis, J.S, Eichenberger, E., Holland, T.L., Fowler, V.G., 2015: Staphylococcus aureus infections: epidemiology, pathophysiology, clinical manifestations, and management. Clin. Microbiol. Rev., 28, 603-661.

14. Zaidi, S., Dahiya, P., 2015: In vitro antimicrobial activity, phytochemical analysis and total phenolic content of essential

\section{Received July 7, 2017}

Accepted September 8, 2017 oil from Mentha spicata and Mentha piperita. International Food Research Journal, 22, 2440-2445.

15. Zaika, L. L., 1988: Spices and herbs: their antibacterial activity and its determination. J. Food Saf., 23, 97-118. 